\title{
Light-controlled drug releasing polymer films combining LbL self-assembly and host-guest interactions
}

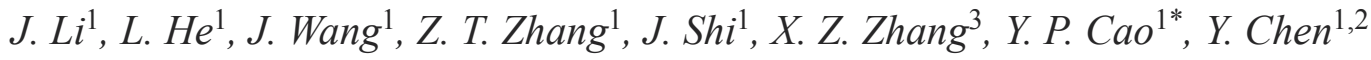 \\ ${ }^{1}$ Institute for Interdisciplinary Research \& Key Laboratory of Optoelectronic Chemical Materials and Devices of Ministry \\ of Education, Jianghan University, 430056 Wuhan, China \\ ${ }^{2}$ Département de Chimie, Ecole Normale Supérieure, 24 Rue Lhomond, F-75231 Paris Cedex 05, France \\ ${ }^{3}$ Key Laboratory of Biomedical Polymers of Ministry of Education \& Department of Chemistry, Wuhan University, \\ 430072 Wuhan, China
}

Received 24 August 2013; accepted in revised form 14 October 2013

\begin{abstract}
By combining LbL (layer-by-layer) self-assembly approach and host-guest interactions, a unique multilayer film was constructed and employed for a light-controlled drug release system. The drug molecules can be loaded and released into the resulting polyelectrolyte multilayers containing azobenzene (Azo) function groups by using the irradiation of visible light and UV light alternately. The photo-sensitivity of the multilayer films was studied through UV-vis spectrum, fluorescence spectrum and confocal microscopy. The target molecules could be rapidly released from the multilayers after $300 \mathrm{~W}$ UV light irradiation for 20 minutes. Moreover, they could be readsorbed into the multilayers uniformly when illuminated under the $300 \mathrm{~W}$ visible light for 10 minutes confirmed by the observation of confocal microscopy, and the readsorption ratio exceeds $100 \%$ evidenced from UV-vis spectroscopy. After several cycles of the above-mentioned process, the multilayer films show good fatigue resistance. All these results indicate the photo-sensitivity and high-efficiency of the multilayer films, which have great potential in controlled drug delivery platform and biomedical applications.
\end{abstract}

Keywords: coatings, layer-by-layer, host-guest interactions, light response, drug delivery

\section{Introduction}

In the past decade, supramolecular chemistry has attracted more and more attention and gradually penetrated into various areas of material and biomedical science [1-3]. The supramolecular system based on host-guest interactions have seen a significant breakthrough, which was shown to be partially successful for drug release [4], biological sensors [5], optically controlled catalytic system [6], molecular shuttles [7], hydrogels [8], and micelles and vesicles [9]. Particularly, photo-controlled molecular recognition of cyclodextrin (CD) with Azobenzenes (Azo) has been widely used for host-guest inclusion compound. Cyclodextrin, a typical host molecule, is a kind of polysaccharides with good biocompatibility and water-solubility. As one of the most widely used guest molecule to cyclodextrin, Azo constitute a class of light-responsive compounds that can undergo trans-cis photoisomerization in response to UV and visible (Vis) light [10, 11]. It is well-known that the apolar and rodlike trans-Azo can form a stable inclusion complex with $\mathrm{CD}$, while the bent and polar cis-Azo cannot. This process is fully reversible under irradiation with UV and Vis light alternately. Reports about the reversible and photo-responsive supramolecular system have come to the fore [6, 12-14]. For example, Zou et al. [15] first put forward supramolecular type amphiphilic molecule concept and prepared a series of rotaxane-like supramolecular amphiphiles. Alter-

\footnotetext{
${ }^{*}$ Corresponding author, e-mail: softmatter@163.com

(C) BME-PT
} 
nating irradiation with UV and Vis light allows for reversible sliding of $\alpha$-CD along the surfactant, leading to the formation of rotaxane-like vesicles. Based on this principle, light-responsive host-guest assembly and disassembly between Azo and CD hold great potential to design a reversible drug delivery profile $[16,17]$.

Layer-by-layer (LbL) self-assembly approach is particularly well-suited for fabricating functional multilayer thin films with ultrafine nanometer-scale structure for use as drug delivery vehicles [18-21]. The traditional means of loading drugs on layers of LbL platform always uses physical adsorption or chemical bonding. However, these methods of loading drugs have a common drawback: drug loading and release is irreversible. This is a critical problem in LbL multilayers for drug delivery. Therefore, it's desired to devise a reversible LbL-based system to manipulate the drug release in a more controllable manner. In the past decade, the pioneering work on LbL assembly with supramolecular interaction has been established by Ikeda et al. [22]. Subsequently, Smith et al. [23] have reported that loading smallmolecule drugs on the layers of LbL-based system using supramolecular interaction. On another front, the light-induced drug release from a LbL-based system is the most elegant way to combine reversibly and efficiency, because light as an attractive stimulus can be applied rapidly, remotely, and locally $[24,25]$. While numerous well-established photoresponsive LbL-based systems are available [26, 27], their applications in drug release based on polyelectrolyte multilayers or coatings is scarce and little progress.

Thus, all mentioned above inspires us to develop a reversible light-controlled drug releasing thin film by combining polyelectrolyte LbL technique and host-guest interactions, which is capable of addressing the demand for small-molecule delivery with highly controlled release kinetics. Our basic approach is to conjugate drugs with $\alpha$-CD through chemical bonding due to the availability of functional groups on their surface. Meanwhile, by introducing the Azo to the layers of LbL system, multiple drugs or functional groups can be loaded into the Azo polymers directly and mildly via host-guest interactions. Thus, $\alpha$-CD-drug complexes will be released and loading from the polyelectrolyte multilayers under alternating irradiation with UV and Vis light. Moreover, the usefulness of cyclodextrin conjugated with drugs offers a simple method to increase drugs solubility, bioavailability, stability. Herein, $\alpha-C D$ modified rhodamine B ( $\alpha$-CD-RhB) was selected as a drug model. We believe that the ability of $\alpha-C D$ to complex with multiple drugs gives these LbL films available versatility to many traditional drug delivery systems [28].

Previously, we have demonstrated that an intelligent 'PnP' (Plug and play) polyanionic template driven by the photo-switchable host-guest interactions was first prepared for the light controlled loading/unloading of small molecule drugs. We have shown that the antineoplastic drug and other functional moieties such as target ligand can be simultaneously released and loaded into the template by using UV and Vis light irradiation alternately [29]. Herein, we describe polyelectrolyte LbL multilayers based on Poly(diallydimethylammonium chloride) (PDAC) and azo-modified polyacrylic acid (PAA- $\mathrm{C}_{6}$-Azo) loaded $\alpha$-CD-RhB via host-guest interactions as a drug carrier that can reversibly capture and release small drugs. From the investigation, $\alpha-\mathrm{CD}-\mathrm{RhB}$ could be rapidly released from the multilayers after $300 \mathrm{~W}$ UV light $(365 \mathrm{~nm})$ irradiation for 20 minutes and they could be adsorbed into the substrate uniformly when illuminated under $300 \mathrm{~W}$ Vis lamp (455 nm) for 10 minutes. Moreover, a 'forever'-pattern was presented onto this LbL film surface via area-selective release and the obtained results may be of great potential for applications in biomedical device based on well-defined nanosized materials surface of arbitrary topography. Light-responsivity of the host-guest interactions based on Azo and $\alpha$-CD-RhB and schematic illustration of $\alpha$-CD-RhB-loading polyelectrolyte multilayers are illustrated in Figure 1. Multilayers are denoted as (PAA- $\mathrm{C}_{6}$-Azo/PDAC)n where $n$ is the number of deposited bilayers.

\section{Experimental details}

\subsection{Materials and characterization}

Acryloyl chloride (AC), rhodamine $\mathrm{B}(\mathrm{RhB})$ and 3aminopropyltriethoxysilane (APTES), PDAC (poly (diallydimethylammonium chloride) solution, $M_{\mathrm{v}}=$ 20 000-35 000, 20\%) were obtained from Shanghai Aladin Co., Ltd. (China) and used directly. N-(3dimethylaminopropyl)-N'-ethylcarbodiimide hydrochloride (EDC.HCl, 98.5\%), 1-azobiscyclo-hexanecarbonitrile $(\mathrm{ABCN}), \mathrm{N}, \mathrm{N}^{\prime}$-dimethylformamide (DMF), 1,4-dioxane and dimethyl sulfoxide (DMSO) 
a)
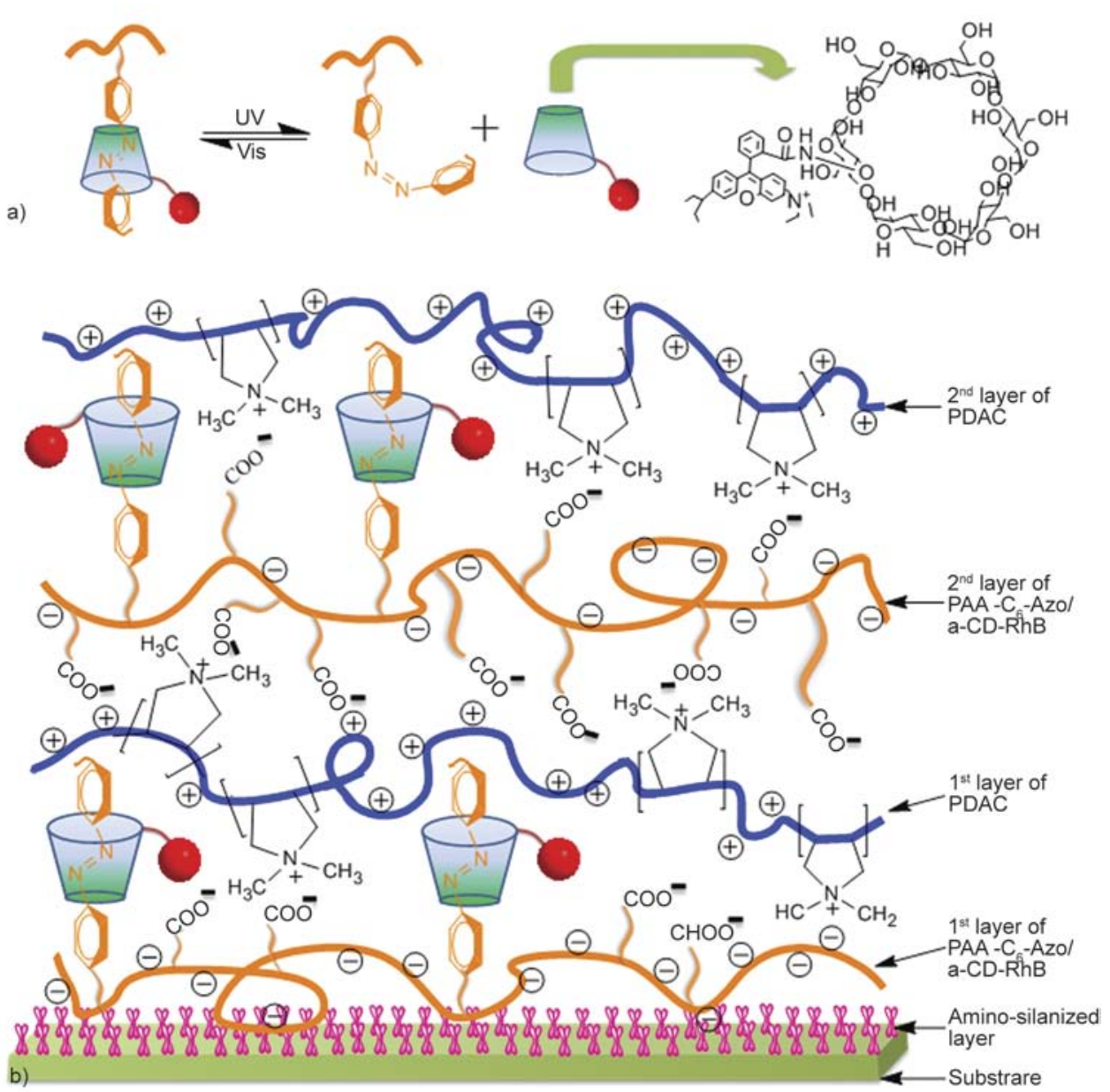

Figure 1. (a) Photoresponse of the host-guest system of Azo group and $\alpha$-CD-RhB. (b) Schmatic representation of polyelectrolyte multilayers loaded with $\alpha-C D-R h B$.

were purchased from Aldrich. DMF was azeotropically distilled with benzene for dehydration and then distilled under vacuum. DMSO and 1,4-dioxane were previously dried with molecular sieves. $\mathrm{ABCN}$ was recrystallized from methanol before use. All other chemicals were used without further purification. All absorption and fluorescence measurements were performed on solutions in $1 \mathrm{~cm}^{2}$ quartz cuvettes. Absorption spectra were measured on a Shimadzu UV-2550 spectrometer. Fluorescence spectra were measured on a Photon Technologies International LS-55 luminescence spectrometer. Solutions were made into $1 \mathrm{mg} / \mathrm{mL}$ aqueous. The fluorescence images of multilayer films before and after UV light irradiation were viewed using confocal laser scanning microscopy with BD Laser at $543 \mathrm{~nm}$.

\subsection{Preparation of poly (acryloyl chloride) (PAC)}

PAC was synthesized according to the literature [30]. Briefly, Acryloyl chloride (20 mL), dry 1,4- dioxane $(20 \mathrm{~mL})$, and $\mathrm{ABCN}(0.668 \mathrm{~g})$ were added into a flask under $\mathrm{N}_{2}$ protection. The flask was sealed and then heated in an oil bath $\left(50^{\circ} \mathrm{C}\right)$ for $14 \mathrm{~h}$. The polymer was precipitated by adding petroleum ether $(100 \mathrm{~mL})$, collected by filtration, and washed twice with petroleum ether. The product was dried at $60^{\circ} \mathrm{C}$ under vacuum for $48 \mathrm{~h}$.

\subsection{Preparation of $\mathrm{PAA}^{-\mathrm{C}_{\mathbf{6}}-\mathrm{Azo}}$}

PAA- $\mathrm{C}_{6}$-Azo was prepared as described elsewhere $[31,32]$. PAC $(0.3 \mathrm{~g}, 0.0033 \mathrm{~mol})$, triethylamine $(0.56 \mathrm{~mL}, 0.0040 \mathrm{~mol})$, and 2-[4-(4-ethoxyphenylazo) phenoxy] ethanol (whose amount was determined by the required degree of functionalization) were dissolved in anhydrous DMF $(33 \mathrm{~mL})$. The mixture was stirred at room temperature for $12 \mathrm{~h}$ under $\mathrm{N}_{2}$ protection. Then suitable amount of water was added into the mixture and stirred for $10 \mathrm{~min}$. The product was precipitated from $\mathrm{HCl}$ water solution $(0.01 \mathrm{~mol} / \mathrm{L})$, collected by filtration, washed several times with water, and dried under vacuum. 


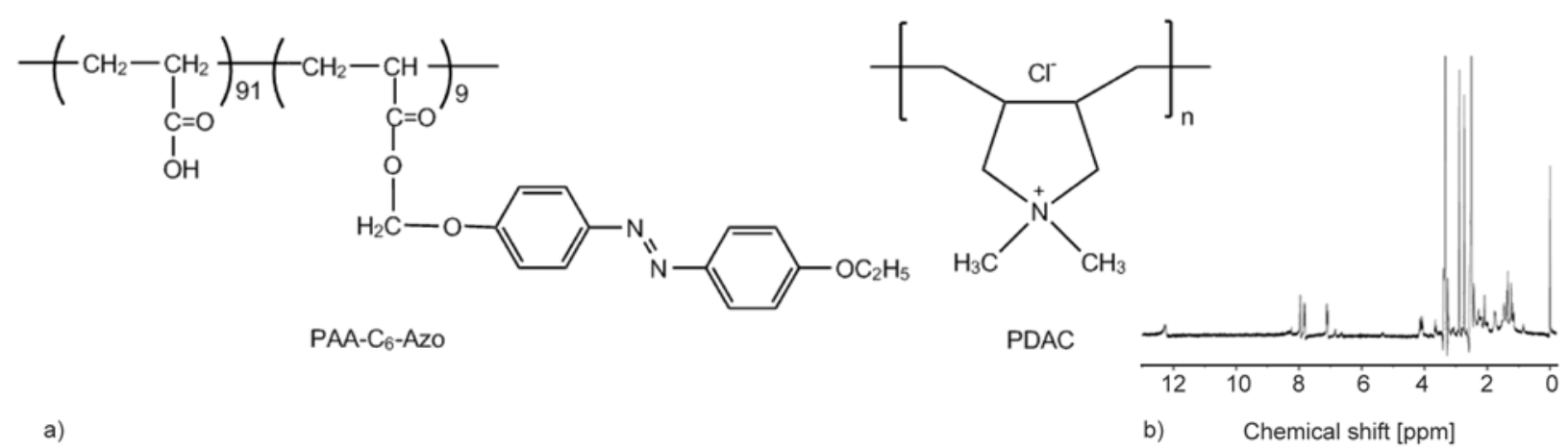

Figure 2. (a) The molecular structure of linear polymer PAA- $\mathrm{C}_{6}-\mathrm{Azo}$ and polypositive charge polymer PDAC. (b) The ${ }^{1} \mathrm{H}$ NMR spectra of PAA-C 6 -Azo.

The polymer was further purified by dissolving in THF and precipitated from petroleum ether, collected by filtration, and washed twice with petroleum ether. The final product was dried at $70^{\circ} \mathrm{C}$ under vacuum for $24 \mathrm{~h}$. The molecular weight of PAA- $\mathrm{C}_{6^{-}}$ Azo was determined by GPC measurement $\left(M_{\mathrm{n}}\right.$ : $\sim 28.6 \mathrm{kDa}$, PDI: 1.98), and the degree of modification (the number of Azo molecules to one acrylic acid unit of PAA) was calculated to be $9 \mathrm{~mol} \%$ by ${ }^{1} \mathrm{H}$ NMR as shown in Figure $2 b$.

\subsection{Synthesis of $\alpha$-CD-rhodamine B $(\alpha-C D-R h B)$}

$\alpha$-CD-RhB was synthesized according to our previous paper [29]. Briefly, $60 \mathrm{mg} 3-\mathrm{NH}_{2}-\alpha-\mathrm{CD}$ and $148 \mathrm{mg} \mathrm{RhB}$ were dissolved in $5 \mathrm{~mL}$ deionized water, after the solution's $\mathrm{pH}$ value was adjusted to 5 , $80 \mathrm{mg}$ EDC was added into this solution and stirred for $24 \mathrm{~h}$ at room temperature. The reaction mixture was poured into a large excess of acetone to recover the product. The residue was washed with acetone four times and dried for 2 days under vacuum drying.

\subsection{Inclusion complex formation}

The drug complex of PAA-C6-Azo/ $\alpha-C D-R h B$ was prepared as follows: $32.2 \mathrm{mg}\left(1.1 \cdot 10^{-3} \mathrm{mmol}\right)$ PAA$\mathrm{C}_{6}$-Azo was respectively dissolved in $40 \mathrm{~mL} \mathrm{H}_{2} \mathrm{O}$ (a suitable amount of sodium hydrogen carbonate was added into the solution to promote the solubility of PAA- $\mathrm{C}_{6}$-Azo). After completely dissolving, the $\mathrm{pH}$ values of the solution were adjusted to be 7.0 by adding a few drops of $\mathrm{HCl}$ dilute solution. Then $40 \mathrm{mg}(0.0286 \mathrm{mmol}) \alpha-\mathrm{CD}-\mathrm{RhB}$ was respectively added under ultrasonic condition at room temperature. The mixtures were stirred overnight at room temperature and then dialysis against uncomplexed $\alpha$-CD-RhB for $48 \mathrm{~h}$ in a dialysis tube.

\subsection{Substrate preparation}

Quartz slides were used as substrates for the UV-vis absorption. A quartz substrate was immersed into a fresh piranha solution $\left(30 \% \mathrm{H}_{2} \mathrm{O}_{2}: 98 \% \mathrm{H}_{2} \mathrm{SO}_{4}(\mathrm{v} / \mathrm{v})\right.$ $=1: 3$; CAUTION: Piranha solution is a very aggressive, corrosive solution, and appropriate safety precautions should be utilized, including the use of acid-resistant gloves and adequate shielding) and heated until no bubbles were released. The substrate was rinsed carefully with deionized water and dried with nitrogen. The cleaned quartz slide was treated in 2\%(v/v) APTES/95\% ethanol solution for $20 \mathrm{~min}$, and then was dehydrated at $110^{\circ} \mathrm{C}$ for $1 \mathrm{~h}$ to obtain the amino-silanized quartz slide.

\subsection{PAA-C 6 -Azo/PDAC multilayer fabrication and characterization}

Linear polymers we used in the experiments are shown in Figure 2a. First, $32.2 \mathrm{mg}\left(1.1 \cdot 10^{-3} \mathrm{mmol}\right)$ PAA- $\mathrm{C}_{6}$-Azo and $80 \mathrm{mg}\left(4.0 \cdot 10^{-4} \mathrm{mmol}\right)$ PDAC were respectively dissolved in $40 \mathrm{~mL} \mathrm{H}_{2} \mathrm{O}$ (a suitable amount of sodium hydrogen carbonate was added into the solution to promote the solubility of PAA- $\mathrm{C}_{6}$-Azo). After completely dissolving, the $\mathrm{pH}$ values of the solution were adjusted to be 7.0 by adding a few drops of $\mathrm{HCl}$ dilute solution. Next, a freshly treated quartz wafer was alternately dipped in the PAA- $\mathrm{C}_{6}$-Azo solution and the PDAC solution each for $10 \mathrm{~min}$. After each dipping, the wafer was washed with enough Milli-Q water for $30 \mathrm{~s}$.

\subsection{The extraction of $\alpha-C D-R h B$ from multilayers}

It has been reported [33] that the release of small molecules from the LbL film through $\mathrm{pH}$ sensitive, ion strength, thermo-sensitive and so on. Here we use the UV light to control the release and uptake of 
the model molecule. The UV irradiating light was from a high-intensity $365 \mathrm{~nm}$ UV lamp equipped with $5 \mathrm{in}$. diameter filter. The intensity of the lamp was $8000 \mu \mathrm{W} / \mathrm{cm}^{2}$ at distance of $15 \mathrm{in}$. A $300 \mathrm{~W}$ xenon lamp equipped with a filter $(\lambda=455 \mathrm{~nm})$ was used as visible light source. The sample was placed $15 \mathrm{~cm}$ away from the lamp. The surrounding temperature of the samples was controlled at $25^{\circ} \mathrm{C}$ using a cold plate.

\subsection{The reversibility of unloading/loading behavior}

To examine the reversibility of unloading/loading behavior, a quartz substrate with 12 assemble monolayers was transferred into a $20.0 \mathrm{~mL}$ water and then irradiated by UV light. After 20 minutes of UV light irradiation, the sample was placed into a cuvette with $20.0 \mathrm{~mL}$ of $\alpha$-CD-RhB aqueous solution $(0.38 \mathrm{mmol})$ and then irradiated by visible light for another 10 minutes. The unloading/loading curve of $\alpha$-CD-RhB at $\lambda=566 \mathrm{~nm}$ was monitored for more than 8 cycles of UV/visible light irradiation.

\section{Results and discussion}

\subsection{The characterization of the host-guest interactions}

UV-vis spectroscopy is a widely applicable means for charactering the formation or dissociation of a host-guest system $[34,35]$. We firstly carried UVvis studies in aqueous solution to understand the inclusion complex of Azo with $\alpha-C D-R h B$, as shown in Figure 3a. Distinct inclusion complex effects on absorption were encountered for dye molecules exhibiting host-guest stoichiometry-dependent shifts in their spectra. In the same concentration $\left(7.8 \cdot 10^{-6} \mathrm{M}\right)$, the maximum absorbance of the

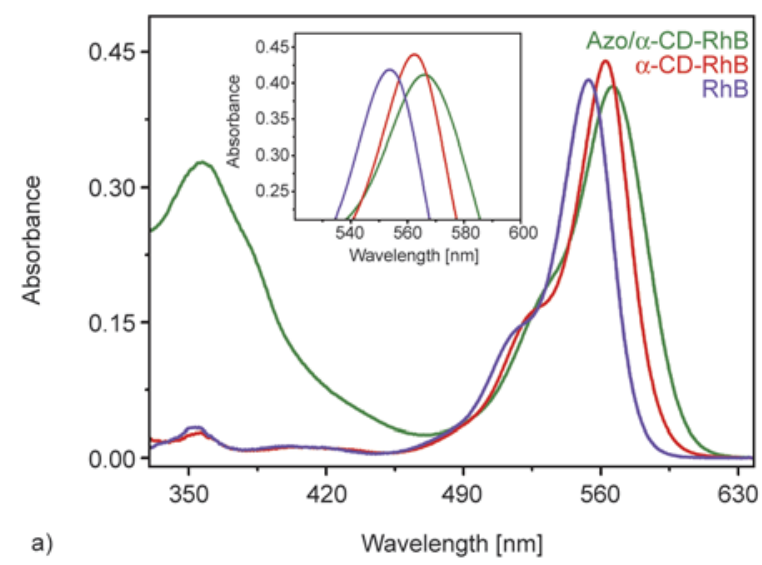

$\mathrm{RhB}, \alpha-\mathrm{CD}-\mathrm{RhB}$ and host-guest system (Azo/CD$\mathrm{RhB}$ ) are located in 552, 563 and $565 \mathrm{~nm}$ respectively, which means that the three solutions tagged by RhB happen red shift gradually. We can observe the difference by comparing the molecular structure of RhB with $\alpha$-CD-RhB. For $\alpha-C D-R h B$, the carboxyl group in $\mathrm{RhB}$ turns into amido linkage, and chromophores are unchanged but the auxochromes vary, leading to the obvious decrease for the energy of the electronic transition. Therefore, the maximum absorbance undergoes red shift. It has been established that the formation of the host-guest system would affect the maximum absorbance as well as the characteristic absorption [36]. With the addition of the guest molecule, the maximum absorption wavelength of the guest molecule (Azo function molecule) is red shifted and its absorbance increases, reflecting that the Azo functional groups have dropped from hydrophilic conditions into the hydrophobic environment. These data provide enough evidence for the formation of supramolecular system based on Azo and $\alpha-\mathrm{CD}$ [37]. In case of the formation of the host-guest interactions, the environment of the chromophoric group has changed and the polarity becomes weaker. As a result, the hydrophobic environment leads to the maximum absorbance red shift as well [38].

Rhodamine B emits strong fluorescence in the specific excitation wavelength $\left(\lambda_{\mathrm{Ex}}=550 \mathrm{~nm}\right)$. This directly provides us with the opportunity of detecting the characteristic emission wavelength of $\mathrm{RhB}$, $\alpha-C D-R h B$ and Azo/ $\alpha-C D-R B$ solution in the same concentration using fluorescence spectrum. Figure $3 \mathrm{~b}$ shows their maximum characteristic emission wavelengths are located in 575,579 and $582 \mathrm{~nm}$,

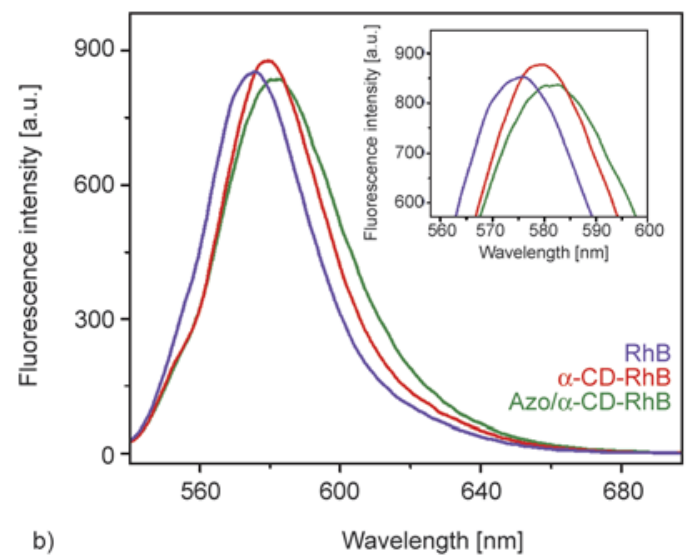

b)

Figure 3. The host-guest interaction characterized by UV-vis spectroscopy (a) and fluorescence spectrophotometer (b). The inset shows the magnification of the characteristic absorption. The violet line is the absorption curve of RhB; the red line is the adsorption of $\alpha-\mathrm{CD}-\mathrm{RhB}$ and the olive one stands for the supramolecular Azo/ $\alpha-\mathrm{CD}-\mathrm{RhB}$. 
indicating gradual red shift for the three solutions. Obviously, the fluorescence spectrum result is consistent well with that of UV-vis spectroscopy. In addition, the result can confirm our conclusion above that the formation of the amido bond change the electronic environment. As described by Wagner, the cause of further red shift for the host-guest system and LbL film should be attributed to the surrounding from aqueous solution state to dry LbL film after nitrogen blow [39]. Overall, although there is no direct proof for formation of a true hostguest inclusion complex, the data observed by fluorescence spectrum, as well as the results from UVvis spectroscopy, does provide compelling indirect evidence that such inclusion complex based Azo and $\alpha-C D-R B$ is indeed formed.

\subsection{Fabrication of multilayer films via electrostatic interaction}

As well-known, electrostatic interaction is the most common driving force to build self-assembly multilayer films [40]. Water-soluble and multiple charge are required for the components to construct the electrostatic LbL multilayers. However, strict matching in position between the charges is not necessary, so it is unavoidable to introduce more than one building components into multilayer film with specific sequence [41]. In our work, photosensitive multilayers were prepared by alternately deposition of polyanion PAA-C6-Azo and polycation PDAC in aqueous. The stepwise self-assembly of the multilayers is characterized by UV-vis spectroscopy, as shown in Figure 4a. The absorbance maximum of

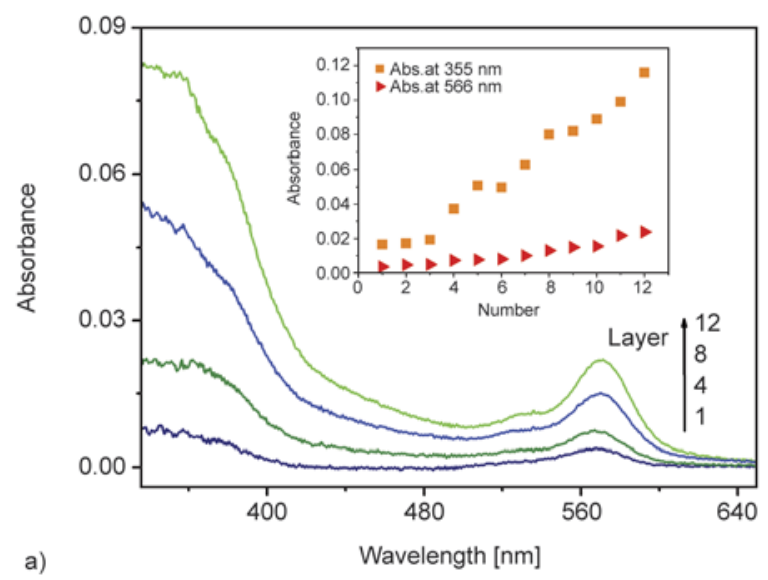

$\alpha-C D-R h B$ in the film at $566 \mathrm{~nm}$ is taken as the reference for monitoring the film growth. A linear increase with the number of layers is observed, which indicates this is a regular deposition process. Additionally, the absorption bands between 300 and $370 \mathrm{~nm}$ is mainly attributed to the $\pi-\pi^{*}$ transition of the Azo groups in PAA- $\mathrm{C}_{6}$-Azo. Moreover, we observe a similar linear relationship between the peak intensity of Azo at $355 \mathrm{~nm}$ and number of bilayers. It should be noted that the $\alpha$-CD-RhB-loading multilayer desorbs abundantly during the deposition of the PDAC layer. After further deposition of an additional PAA- $\mathrm{C}_{6}-\mathrm{Azo} / \alpha-\mathrm{CD}-\mathrm{RhB}$ layer, the characteristic absorbance of $\alpha-\mathrm{CD}-\mathrm{RhB}$ increase again. A possible explanation for this phenomenon could be related to the ionic strength of the PDAC solution $[28,42]$.

We have further investigated the self-assembly process from fluorescence spectrum (Figure 4b). It is clear seen that quartz/multilayers as well as solution of the host-guest system emit characteristic fluorescence, which are similar to $\mathrm{RhB}$, whereas quartz without self-assembly are not fluorescent $\left(\lambda_{\mathrm{Ex}}=\right.$ $550 \mathrm{~nm}$ ). This confirms $\alpha$-CD-RhB-loading linear polymers have been successfully assembled on the surface of quartz wafers. Further, as the number of layers increases, the fluorescence intensity for the quartz slide also grows (data not shown). These results are in good agreement with that of UV-vis spectroscopy. However, in comparison with the solution of the supramolecular system, the characteristic emission peak for Azo/ $\alpha-\mathrm{CD}-\mathrm{RB}$ has moved from 580 to $587 \mathrm{~nm}$. The reason for this red shift

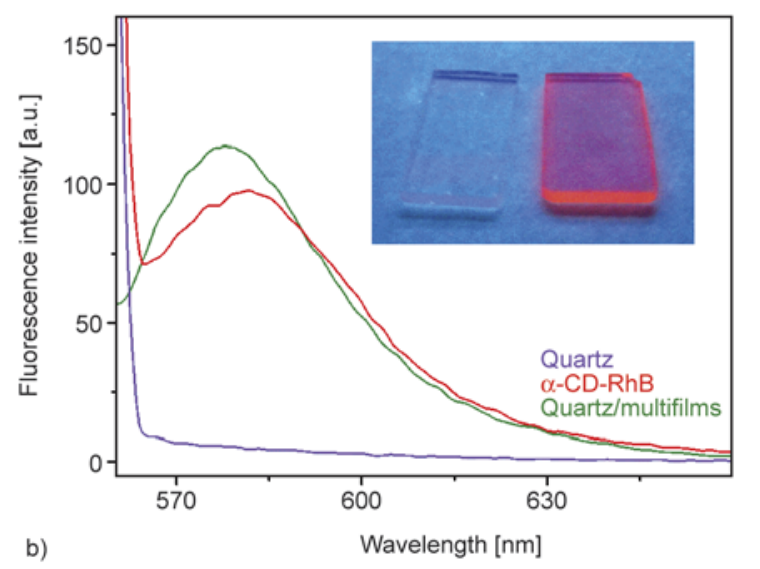

Figure 4. (a) The multilayers assembled on a quartz substrate and measured after each deposition treatment. The inset scatter diagram shows growth tendency of Azo (orange) and RhB (red), respectively. (b) Fluorescence spectra of Quartz, $\alpha$-CD-RB solution and Quartz/multilayers $(\alpha$-CD-RhB-loading 8-bilayer multilayers assembled on the quartz slide). The inset shows the photographs of a quartz slide before (left) and after (right) assembly treatment under a $28 \mathrm{~W}$ UV light $(365 \mathrm{~nm})$ in a dark environment. 
may be the change of surrounding environment from dryness to aqueous, so does the polarity [43, 44].

\subsection{Light-triggered release and loading}

The multilayers were observed with confocal laser scanning microscopy (CLSM) (Nikon C1-si, BD Laser at $543 \mathrm{~nm}$ ). It is clear seen that the red fluorescent surface of the (PAA- $\left.\mathrm{C}_{6}-\mathrm{Azo} / \mathrm{PDAC}\right)_{8}$ film confirmed $\alpha-\mathrm{CD}-\mathrm{RhB}$ is loaded on the layers before the irradiation of the UV light (Figure 5a). While the fluorescence almost disappears after irradiation with UV light $(365 \mathrm{~nm})$ for 20 minutes, indicating the release of $\alpha-C D-R h B$ from the films (Figure $5 b$ ). Before release, an average surface coverage of 0.91 drug molecules per $\mathrm{nm}^{2}$ is calculated from the BeerLambert law, while the value drops below 0.05 after release. This reveals that the release process is com-

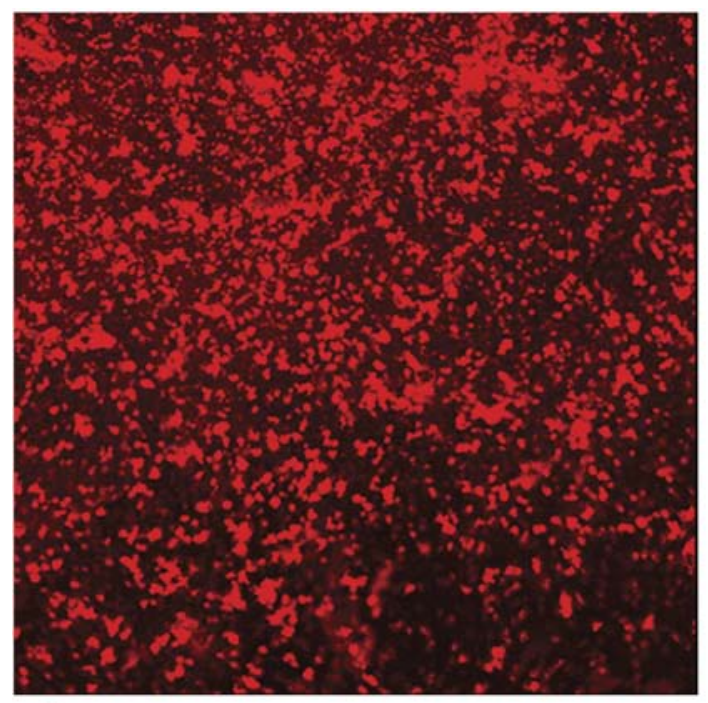

a)

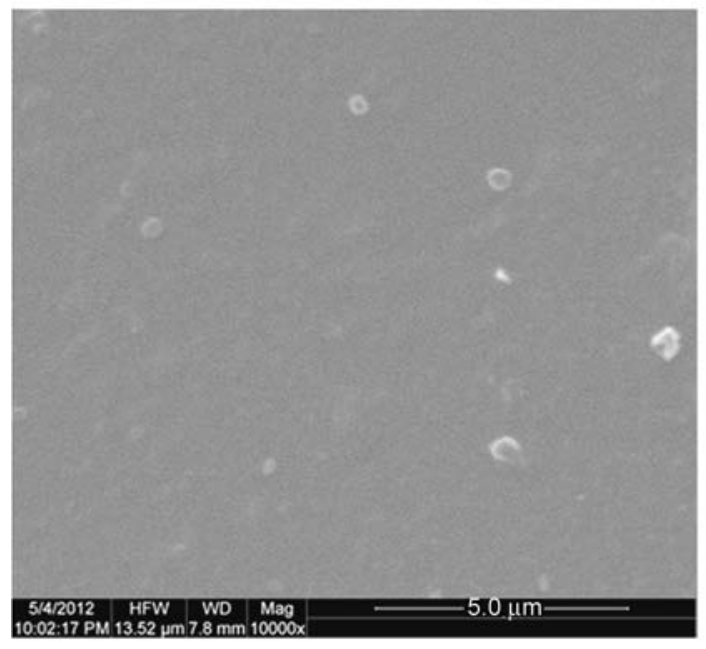

b) plete and obvious, which is attributed to disassemble of the specific host-guest interactions between Azo and $\alpha$-CD-RhB upon UV irradiation.

Further, the surface morphology of multilayer films has been characterized by scanning electron microscopy (SEM). The SEM image of the $\alpha$-CD-RhBloading 8-bilayer film shown in Figure 5b illustrates a coarse surface morphology and some massive substance is observed, which may be the results of uniform assembly. After UV light irradiation, the $\alpha$-CD-RhB-released multilayer shows a smooth and uniform surface coverage. We assume the irregular topography surface is a result of the fast assembly process. Therefore, the linear Azo polymers get behind in adjusting their configurations, but it definitely deserves further investigation, which is outside of the scope of this work. After illumination of
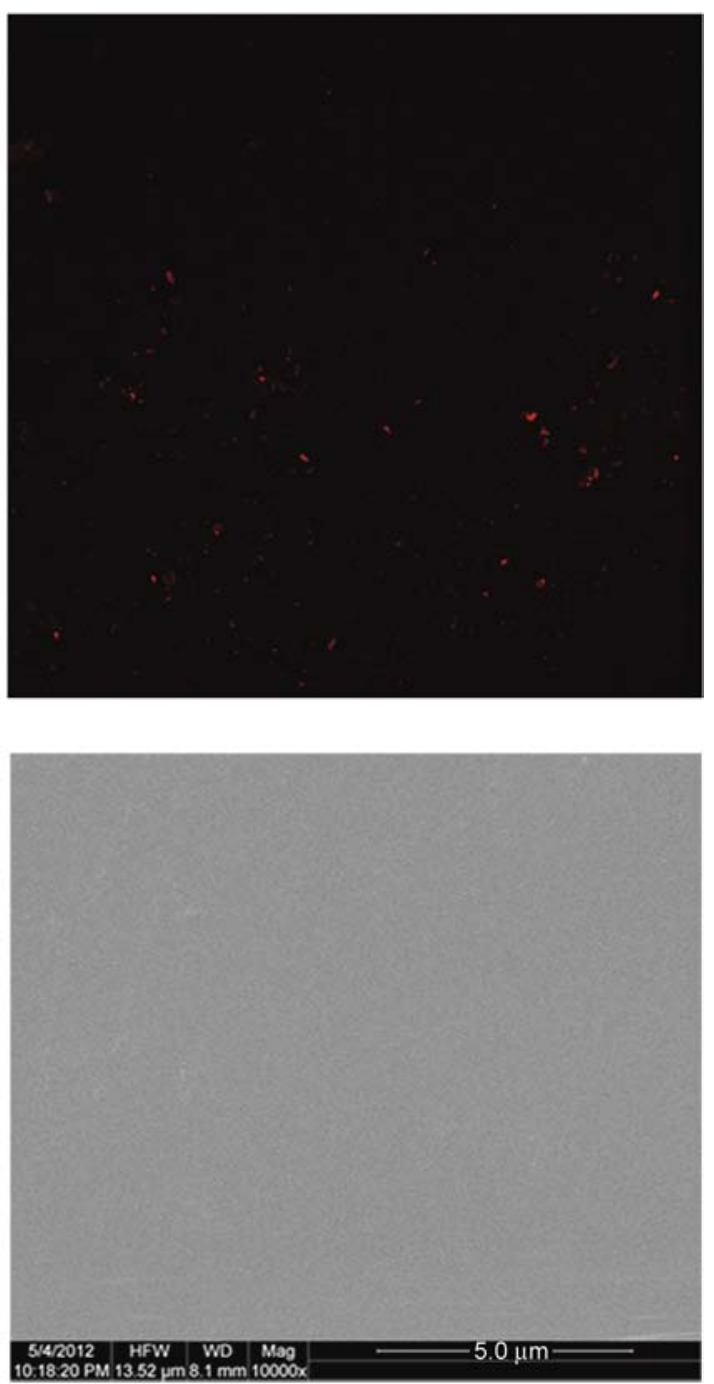

Figure 5. (a) CLSM images of multilayer films before (left) and after (rihgt) UV irradiation (the scale bar is $100 \mu \mathrm{m}$ ).

(b) SEM images of multilayers before (left) and after (right) UV irradiation (the scale bar is $5 \mu \mathrm{m}$ ). 


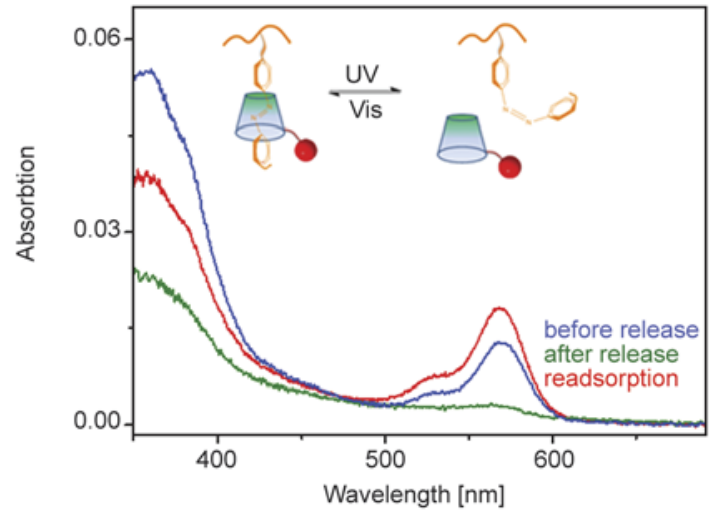

Figure 6. Absorption spectra of the $\alpha$-CD-RhB loading multilayers before and after UV light irradiation for 20 minutes, then Vis light irradiation for 10 minutes in $40 \mathrm{~mL} \alpha-\mathrm{CD}-\mathrm{RhB}$ aqueous solution for loading $(1 \mathrm{mg} / \mathrm{mL})$.

the UV light, the photoisomerization of the Azo resulted in the release of $\alpha-\mathrm{CD}-\mathrm{RhB}$ from the film, then the surface become smooth and even.

UV-vis spectrum was employed to monitor the release and reloading of multilayers (Figure 6). Application of 20 min UV irradiation induces a clear and rapid loss of dye from the 8-bilayer film. The absorbance of multilayer at $568 \mathrm{~nm}$ is about 0.026 before release, while the absorbance almost disappears after UV light treatment. It can be concluded that the loaded $\alpha-\mathrm{CD}-\mathrm{RhB}$ is almost completely washed out. For reloading studies, the film was immersed into $1 \mathrm{mg} / \mathrm{mL}$ of $\alpha$-CD-RhB solutions for $10 \mathrm{~min}$ Vis light $(455 \mathrm{~nm})$ irradiation. Surprisingly, irradiation of Vis light for the multilayers after released introduces much more drug molecules move back into the multilayers. This is likely to be caused by a small number of free Azo molecules without assembling with $\alpha-\mathrm{CD}-\mathrm{RhB}$ during the fabrication of multilayers [32]. It should be mentioned that drugs release rate for $\mathrm{PAA}-\mathrm{C}_{6}$-Azo/PDAC film is rapid. $98 \mathrm{wt} \%$ of $\alpha-\mathrm{CD}-\mathrm{RhB}$ is released within 50 min for 20-bilayer film under UV irradiation, while 10-bilayer film takes $28 \mathrm{~min}$ to release of ca. $99 \%$ drugs. Hence, it is difficult to get detailed release rate, but the release trends can be attained. For 20-bilayer film, after 6 min of UV light irradiation, the release percentage starts to increase sharply, which raises to ca. $50 \%$ after $22 \mathrm{~min} .88 \%$ of the loaded drugs is released in 40 min under UV irradiation and it reaches ca. $98 \%$ in $50 \mathrm{~min}$. These results strongly demonstrate that the film exhibits a rapid response for light-triggered release.

\subsection{Reversible loading and release}

In order to investigate the potential of the $\alpha$-CDRhB-loading multilayers as a reversible light-triggered release platform, a quartz substrate with 8 assembled bilayers was transferred into $20.0 \mathrm{~mL}$ water and then irradiated by UV light. After 20 minutes of UV light irradiation, the sample was placed into a cuvette with $20.0 \mathrm{~mL}$ of $\alpha$-CD-RhB aqueous solution $(0.38 \mathrm{mM})$ and then irradiated by visible light for 10 minutes. The absorbance of $\left(\mathrm{PAA}^{-\mathrm{C}_{6}}{ }^{-}\right.$ Azo/PDAC $)_{6}$ film was recorded, as shown in Figure 7. As expected, the multilayers exhibit good photo-responsive properties. It is shown that the absorption efficiency of the PAA-Azo/PDAC film is almost unchanged after 16 times of release and loading under irradiation with UV and Vis light alternately. This result demonstrates that the film is stable in these loading and release conditions, and can be used for a reversible drug delivery system.

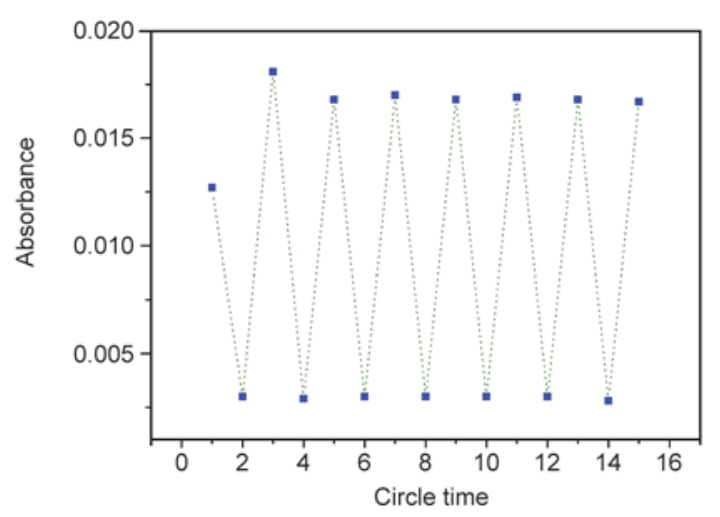

Figure 7. The cyclic absorbance features of $\left(\mathrm{PAA}^{-\mathrm{C}_{6}}\right.$ $\mathrm{Azo} / \mathrm{PDAC})_{6}$ multilayer film at $566 \mathrm{~nm}$ as a result of the cyclic loading and release.

\subsection{Surface patterning}

Finally, to further verify the ability of light-sensitive of the LbL deliver platform, we presented a pattern onto the LbL film surface via area-selective release. Demonstration of the LbL film in areaselective release was conducted using a 'forever'shaped mask (Figure $8 \mathrm{~b}$ ). The procedures of pattern formation are described as follows. Briefly, a quartz slide with as-prepared (PAA- $\mathrm{C}_{6}$-Azo/PDAC) 20 film was transferred into a cuvette with $20.0 \mathrm{~mL}$ deionized water at room temperature. Next, a 'forever'shaped mask was also immersed in water and positioned on the film. Then, they were irradiated by UV light for 40 minutes, followed by a brief wash- 


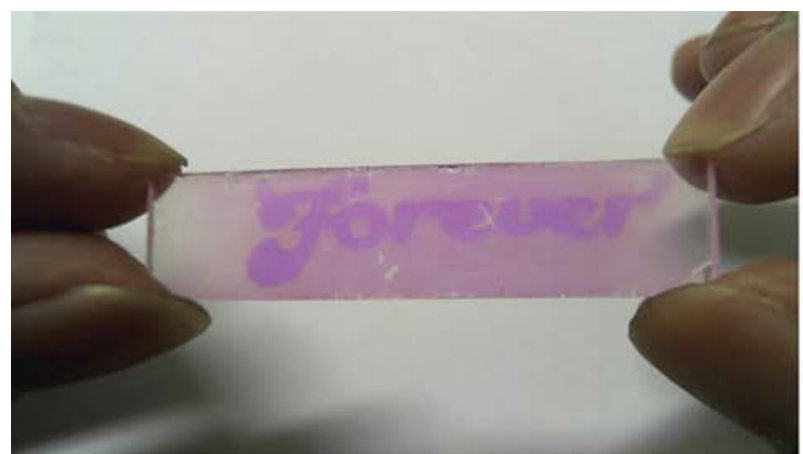

a)

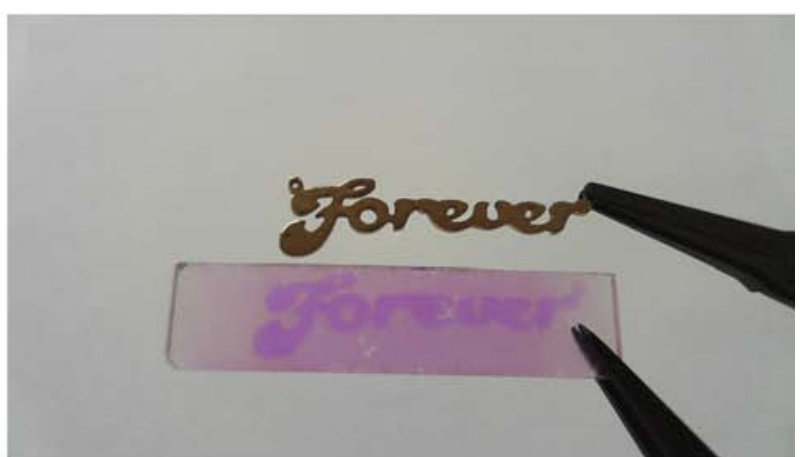

b)

Figure 8. The patterned photograph of (PAA- $\left.\mathrm{C}_{6}-\mathrm{Azo} / \mathrm{PDAC}\right)_{20}$ multilayer film as a result of local release. (a) The well-patterned 'forever' on a quartz slide. (b) The contrast between the mask and the resulting pattern.

ing with neutral water and drying under a nitrogen stream. This process yielded a fluorescent display of 'forever'-shaped pattern. As seen clearly in Figure 8a, The UV irradiation areas are almost colourless, while the UV-free parts are red, indicating the area-selective release of $\alpha-\mathrm{CD}-\mathrm{RhB}$ from the LbL film. The well-patterned 'forever' is distinct with a clearly outline, which attributes to the photo-sensitivity of the multilayer film for release.

\section{Conclusions}

In summary, we have demonstrated a polyelectrolyte polymer film by combining LbL self-assembly and host-guest interactions for light-controlled drug release. The multilayer film based on electrostatic interactions between PDAC and PAA- $\mathrm{C}_{6}$-Azo showed high stability under neutral conditions. Compared with traditional drug-loading methods of using physical uptake and chemical bonding, our supramolecular drug-loading approach of the LbLbased film exhibits convenient drug loading, ideal bonding strength, and light-controlled drug release. Moreover, the resulting film can catch and release multidrug, or other functional groups, as exemplified here by loading and release of $\alpha-\mathrm{CD}-\mathrm{RhB}$. The process for loading and release is reversible for at least eight cycles investigated so far. Equipped with these smart features, this LbL-based film affords useful information for the development of intelligent drug delivery platforms and appropriate biological studies. Currently we are planning to apply this LbL-based system for the light-controlled release in biodegradable poly(lactic acid), which provides an ideal substrate for therapeutic delivery using surgical implantion.

\section{Acknowledgements}

We thank Chinese Key Basic Research Program (2010CB529906), Wuhan Key Technologies R\&D Program (201250499145-10, 2013011001010483), and the Opening Project of Key Laboratory of Optoelectronic Chemical Materials and Devices of Ministry of Education (JDGD2013-16) for funding. One of the authors wish to thank CongCong Ni for helping in UV-vis measurement.

\section{References}

[1] Zhou S-L., Matsumoto S., Tian H-D., Yamane H., Ojida A., Kiyonaka S., Hamachi I.: pH-responsive shrinkage/swelling of a supramolecular hydrogel composed of two small amphiphilic molecules. Chemistry A European Journal, 11, 1130-1136 (2005).

DOI: $10.1002 /$ chem.200400677

[2] Zhao Y., Yokoi H., Tanaka M., Kinoshita T., Tan T.: Self-assembled $\mathrm{pH}$-responsive hydrogels composed of the RATEA16 peptide. Biomacromolecules, 9, 15111518 (2008). DOI: $10.1021 / \mathrm{bm} 701143 \mathrm{~g}$

[3] Ge Z., Xu J., Hu J., ZhangY., Liu S.: Synthesis and supramolecular self-assembly of stimuli-responsive water-soluble Janus-type heteroarm star copolymers. Soft Matter, 5, 3932-3939 (2009).

DOI: $10.1039 / \mathrm{B} 907906 \mathrm{H}$

[4] Isenbügel K., Gehrke Y., Ritter H.: Photo-switchable behavior of azobenzene-dye-modified silica nanoparticles and their assembly with cyclodextrin derivatives. Macromolecular Chemistry and Physics, 213, 227-233 (2012).

DOI: $10.1002 / \mathrm{macp} .201100601$

[5] Sun T., Qing G.: Biomimetic smart interface materials for biological applications. Advanced Materials, 23, H57-H77 (2011). DOI: $10.1002 / \mathrm{adma} .201004326$

[6] Zhang X., Wang C.: Supramolecular amphiphiles. Chemical Society Reviews, 40, 94-101 (2011). DOI: $10.1039 / \mathrm{b} 919678 \mathrm{c}$ 
[7] Wenz G., Han B-H., Müller A.: Cyclodextrin rotaxanes and polyrotaxanes. Chemical Reviews, 106, 782 817 (2006).

DOI: $10.1021 / \mathrm{cr} 970027+$

[8] Peng K., Tomatsu I., Kros A.: Light controlled protein release from a supramolecular hydrogel. Chemical Communications, 46, 4094-4096 (2010).

DOI: $10.1039 / \mathrm{c} 002565 \mathrm{~h}$

[9] Zou J., Guan B., Liao X., Jiang M., Tao F.: Dual reversible self-assembly of PNIPAM-based amphiphiles formed by inclusion complexation. Macromolecules, 42, 7465-7473 (2009).

DOI: $10.1021 / \mathrm{ma} 901276 \mathrm{c}$

[10] Chen C-J., Liu G-Y., Liu X-S., Li D-D., Ji J.: Construction of photo-responsive micelles from azobenzene-modified hyperbranched polyphosphates and study of their reversible self-assembly and disassembly behaviours. New Journal of Chemistry, 36, 694-701 (2012).

DOI: $10.1039 / \mathrm{c} 2$ nj20882b

[11] Ercole F., Davis T. P., Evans R. A.: Photo-responsive systems and biomaterials: Photochromic polymers, light-triggered self-assembly, surface modification, fluorescence modulation and beyond. Polymer Chemistry, 1, 37-54 (2010).

DOI: $10.1039 / \mathrm{b} 9 \mathrm{py} 00300 \mathrm{~b}$

[12] Such G., Evans R. A., Yee L. H., Davis T. P.: Factors influencing photochromism of spiro-compounds within polymeric matrices. Journal of Macromolecular Science Part C: Polymer Reviews, 43, 547-579 (2003). DOI: $10.1081 / \mathrm{mc}-120025978$

[13] Chen L., Xu S., Li J.: Recent advances in molecular imprinting technology: Current status, challenges and highlighted applications. Chemical Society Reviews, 40, 2922-2942 (2011).

DOI: $10.1039 / \mathrm{C} 0 \mathrm{CS} 00084 \mathrm{~A}$

[14] Lee S-W., Takahara N., Korposh S., Yang D-H., Toko K., Kunitake T.: Nanoassembled thin film gas sensors. III. Sensitive detection of amine odors using $\mathrm{TiO}_{2} /$ poly (acrylic acid) ultrathin film quartz crystal microbalance sensors. Analytical Chemistry, 82, 2228-2236 (2010).

DOI: $10.1021 / \mathrm{ac} 901813 \mathrm{q}$

[15] Zou J., Tao F., Jiang M.: Optical switching of selfassembly and disassembly of noncovalently connected amphiphiles. Langmuir, 23, 12791-12794 (2007). DOI: $10.1021 / 1 \mathrm{a} 702815 \mathrm{~h}$

[16] Dsouza R. N., Pischel U., Nau W. M.: Fluorescent dyes and their supramolecular host/guest complexes with macrocycles in aqueous solution. Chemical Reviews, 111, 7941-7980 (2011). DOI: $10.1021 / \mathrm{cr} 200213 \mathrm{~s}$

[17] Ferris D. P., Zhao Y-L., Khashab N. M., Khatib H. A., Stoddart J. F., Zink J. I.: Light-operated mechanized nanoparticles. Journal of the American Chemical Society, 131, 1686-1688 (2009).

DOI: $10.1021 /$ ja807798g
[18] Hu X., Ji J.: Covalent layer-by-layer assembly of hyperbranched polyether and polyethyleneimine: Multilayer films providing possibilities for surface functionalization and local drug delivery. Biomacromolecules, 12, 4264-4271 (2011). DOI: $10.1021 / \mathrm{bm} 201137 \mathrm{x}$

[19] Tong W., Song X., Gao C.: Layer-by-layer assembly of microcapsules and their biomedical applications. Chemical Society Reviews, 41, 6103-6124 (2012).

DOI: $10.1039 / \mathrm{c} 2 \mathrm{cs} 35088 \mathrm{~b}$

[20] Mohanta V., Madras G., Patil S.: Layer-by-layer assembled thin film of albumin nanoparticles for delivery of doxorubicin. The Journal of Physical Chemistry C, 116, 5333-5341 (2012).

DOI: $10.1021 / \mathrm{jp} 209479 \mathrm{n}$

[21] Ariga K., Ji Q., Hill J. P., Bando Y., Aono M.: Forming nanomaterials as layered functional structures toward materials nanoarchitectonics. NPG Asia Materials, 4, e17/1-e17/11 (2012).

DOI: $10.1038 / \mathrm{am} .2012 .30$

[22] Ikeda A., Hatano T., Shinkai S., Akiyama T., Yamada S.: Efficient photocurrent generation in novel selfassembled multilayers comprised of [60]fullerenecationic homooxacalix[3] arene inclusion complex and anionic porphyrin polymer. Journal of the American Chemical Society, 123, 4855-4856 (2001).

DOI: $10.1021 / \mathrm{ja} 015596 \mathrm{k}$

[23] Smith R. C., Riollano M., Leung A., Hammond P. T.: Layer-by-layer platform technology for small-molecule delivery. Angewandte Chemie International Edition, 48, 8974-8977 (2009).

DOI: $10.1002 /$ anie.200902782

[24] Mei X., Yang S., Chen D., Li N., Li H., Xu Q., Ge J., $\mathrm{Lu}$ J.: Light-triggered reversible assemblies of azobenzene-containing amphiphilic copolymer with $\beta$-cyclodextrin-modified hollow mesoporous silica nanoparticles for controlled drug release. Chemical Communications, 48, 10010-10012 (2012). DOI: $10.1039 / \mathrm{c} 2 \mathrm{cc} 33995 \mathrm{a}$

[25] Wu Y., Wu S., Tian X., Wang X., Wu W., Zou G., Zhang Q.: Photoinduced reversible gel-sol transitions of dicholesterol-linked azobenzene derivatives through breaking and reforming of van der Waals interactions. Soft Matter, 7, 716-721 (2011). DOI: 10.1039/COSM00330A

[26] Gong C. B., Lam M. H-W., Yu H. X.: The fabrication of a photoresponsive molecularly imprinted polymer for the photoregulated uptake and release of caffeine. Advanced Functional Materials, 16, 1759-1767 (2006). DOI: $10.1002 / \mathrm{adfm} .200500907$

[27] Wan P., Jiang Y., Wang Y., Wang Z., Zhang X.: Tuning surface wettability through photocontrolled reversible molecular shuttle. Chemical Communications, 2008, 5710-5712 (2008).

DOI: $10.1039 / \mathrm{b} 811729 \mathrm{~b}$ 
[28] Davis M. E., Brewster M. E.: Cyclodextrin-based pharmaceutics: Past, present and future. Nature Reviews, Drug Discovery, 3, 1023-1035 (2004).

DOI: $10.1038 / \mathrm{nrd} 1576$

[29] Xiao W., Chen W-H., Xu X-D., Li C., Zhang J., Zhuo R-X., Zhang X-Z.: Design of a cellular-uptake-shielding 'plug and play' template for photo controllable drug release. Advanced Materials, 23, 3526-3530 (2011). DOI: $10.1002 /$ adma.201101806

[30] Yang Y. S., Qi G. R., Qian J. W., Yang S. L.: Acryloyl chloride polymer. Journal of Applied Polymer Science, 68, 665-670 (1998).

DOI: $10.1002 /($ sici)1097-4628(19980425)68:4<665::aidapp $18>3.0 . c 0 ; 2-q$

[31] Wu L., Tuo X., Cheng H., Chen Z., Wang X.: Synthesis, photoresponsive behavior, and self-assembly of poly(acrylic acid)-based azo polyelectrolytes. Macromolecules, 34, 8005-8013 (2001).

DOI: $10.1021 / \mathrm{ma} 002215 \mathrm{z}$

[32] Wu M., Cao Y., Zhang X., Zhang Y., Chen Y., He L., Qian Z.: Double 'plug and play' templates technology for photo controllable drug release polyelectrolyte multilayers. Chemical Communications, 48, 98469848 (2012).

DOI: $10.1039 / \mathrm{c} 2 \mathrm{cc} 35274 \mathrm{e}$

[33] Becker A. L., Johnston A. P. R., Caruso F.: Layer-bylayer-assembled capsules and films for therapeutic delivery. Small, 6, 1836-1852 (2010).

DOI: $10.1002 /$ smll.201000379

[34] Ferreira I. R., Ando R. A.: Shifting the azo-hydrazone tautomeric equilibrium of methyl yellow in acidic medium by the formation of inclusion complexes with cyclodextrins. Chemical Physics Letters, 522, 51-53 (2012).

DOI: 10.1016/j.cplett.2011.12.011

[35] Chen Z., Dong S., Zhong C., Zhang Z., Niu L., Li Z., Zhang F.: Photoswitching of the third-order nonlinear optical properties of azobenzene-containing phthalocyanines based on reversible host-guest interactions. Journal of Photochemistry and Photobiology A: Chemistry, 206, 213-219 (2009)

DOI: $10.1016 /$ j.jphotochem.2009.07.005

[36] Park J. W., Song H. J.: Association of anionic surfactants with .beta.-cyclodextrin: Fluorescence-probed studies on the 1:1 and 1:2 complexation. The Journal of Physical Chemistry, 93, 6454-6458 (1989).

DOI: $\underline{10.1021 / \mathrm{j} 100354 \mathrm{a} 035}$
[37] Choudhury S. D., Mohanty J., Upadhyaya H. P., Bhasikuttan A. C., Pal H.: Photophysical studies on the noncovalent interaction of thioflavin $\mathrm{T}$ with cucurbit $[n]$ uril macrocycles. The Journal of Physical Chemistry B, 113, 1891-1898 (2009).

DOI: $10.1021 / j p 8103062$

[38] Fedorova O. A., Chernikova E. Y., Fedorov Y. V., Gulakova E. N., Peregudov A. S., Lyssenko K. A., Jonusauskas G., Isaacs L.: Cucurbit[7]uril complexes of crown-ether derived styryl and (bis)styryl dyes. The Journal of Physical Chemistry B, 113, 10149-10158 (2009).

DOI: $10.1021 / j p 903289 q$

[39] Wagner B. D., Fitzpatrick S. J., Gill M. A., MacRae A. I., Stojanovic N.: A fluorescent host-guest complex of cucurbituril in solution: A molecular Jack O'Lantern. Canadian Journal of Chemistry, 79, 1101-1104 (2001). DOI: $10.1139 / \mathrm{v} 01-094$

[40] Yang S. Y., Rubner M. F.: Micropatterning of polymer thin films with $\mathrm{pH}$-sensitive and cross-linkable hydrogen-bonded polyelectrolyte multilayers. Journal of the American Chemical Society, 124, 2100-2101 (2002). DOI: $10.1021 / \mathrm{ja} 017681 \mathrm{y}$

[41] Matsusaki M., Ajiro H., Kida T., Serizawa T., Akashi M.: Layer-by-layer assembly through weak interactions and their biomedical applications. Advanced Materials, 24, 454-474 (2012). DOI: $10.1002 / \mathrm{adma} .201103698$

[42] Song X., Perlstein J., Whitten D. G.: Supramolecular aggregates of azobenzene phospholipids and related compounds in bilayer assemblies and other microheterogeneous media: Structure, properties, and photoreactivity ${ }^{1}$. Journal of the American Chemical Society, 119, 9144-9159 (1997). DOI: $10.1021 /$ ja971291n

[43] Chakraborty B., Basu S.: Deciphering the host-guest chemistry of acridine yellow and cucurbit[7]uril: An integrated spectroscopic and calorimetric study. Chemical Physics Letters, 507, 74-79 (2011). DOI: 10.1016/j.cplett.2011.03.014

[44] Zhang Y., Sun X-Y., Liu B.: Fluorescent recognition for single- and double-stranded oligonucleotides based on rhodamine B-modified self-assembled bilayers. Chinese Journal of Analytical Chemistry, 37, 665-670 (2009).

DOI: $10.1016 / \mathrm{s} 1872-2040(08) 60103-6$ 